\title{
Funktionale Polymere für ionenselektive Elektroden
}

\author{
Johannes Schwarz ${ }^{1)}$, Kathrin Trommer ${ }^{1)}$, Dietmar Appelhans ${ }^{2)}$, Jörg Kluge ${ }^{2)}$, Brigitte Voit ${ }^{2)}$, \\ Michael Mertig ${ }^{1)}$ \\ ${ }^{1)}$ Kurt-Schwabe-Institut für Mess- und Sensortechnik e. V. Meinsberg, \\ Kurt-Schwabe-Straße 4, 04736 Waldheim, Deutschland \\ ${ }^{2)}$ Leibniz-Institut für Polymerforschung Dresden e.V. \\ Hohe Straße 6, 01069 Dresden, Deutschland
}

Keywords:, All-Solid-State-Elektroden, ionenselektive Membran, Glycopolymere, Calixarene, Polyethylenimine,

\begin{abstract}
Einleitung
Die Nachfrage nach einfach bedienbaren, preisgünstigen und für den Feldeinsatz geeigneten potentiometrischen Sensoren nimmt stetig zu. Für den Praxiseinsatz eignen sich siebdruckgefertigte, miniaturisierbare Analysatoren ohne Flüssigkomponenten, die mechanisch stabil sind und einen geringen Chemikalieneinsatz erfordern [1-5].

Ziel der Arbeit ist die Entwicklung neuer kationenselektiver, potentiometrischer AllSolid-State-Elektroden zur Detektion von $\mathrm{Pb}^{2+}$, $\mathrm{Cd}^{2+}, \mathrm{NH}_{4}{ }^{+}$und $\mathrm{Ca}^{2+}$. Durch Einbindung neuer Rezeptoren bzw. Ionophore in polymerhaltige Matrices sind ionensensitive Elektroden mit dünnen funktionalen Polymerfilmen zu entwickeln. Dendritische Polymere mit hochspezifischen Oberflächen, sowie darin immobilisierte, kationenselektive lonophore, vorzugsweise mit kationenselektiven Calixaren-Strukturen sollen zum Einsatz kommen. Die Beschichtung der graphithaltigen Transducer mit neuen innovativen Sensormaterialien für die Kationen-Analytik steht im Vordergrund des Vorhabens.
\end{abstract}

\footnotetext{
Elektrodenbauweise und-herstellung

Graphithaltige Transducer wurden auf Keramiksubstrate der Abmessungen $50 \times 6$ $\mathrm{mm}$ bzw. 35 × $6 \mathrm{~mm}$ mittels Siebdrucktechnik aufgebracht. Anschließend erfolgte die Abscheidung dünner Polypyrrol-Filme durch Elektropolymerisation auf die Graphitoberflächen und danach das Auftropfen des ionenselektiven Membrancocktails auf das leitfähige Polymer. Die Membranzusammensetzung wurde für die jeweiligen Ionen optimiert. Der Elektrodenaufbau aus mehreren funktionalen Schichten ermöglicht eine reproduzierbare Elektrodenherstellung und eine große Variabilität im Elektrodendesign und Layout. Siebdruckgefertigte Referenzelektroden auf Basis von $\mathrm{AgCl}$-haltigen Pasten wurden entwickelt.
}

Untersuchungen zur Modifizierung der Referenzelektroden mit definierten Elektrolyten erfolgen gegenwärtig.

\section{Ergebnisse}

Neue miniaturisierte, kationenselektive Dickschichtelektroden wurden für potentiometrische lonenbestimmungen entwickelt. Die Elektrodengrundkörper wurden mittels Siebdruck hergestellt. Durch spezifische Modifizierungen der Elektrodenoberflächen mit Polymeren mit immobilisierten, spezifischen Rezeptoren sind empfindliche lonen-Analysatoren für Anwendungen im Umweltbereich realisierbar. Die funktionalen polymerhaltigen Schichten zeichnen sich durch eine gute Haftung und hohe mechanische Stabilität aus.

Abbildung 1 zeigt einen ionenselektiven AllSolid-State-Sensor mit integrierter Referenzelektrode auf $\mathrm{Ag} / \mathrm{AgCl}$-Basis.

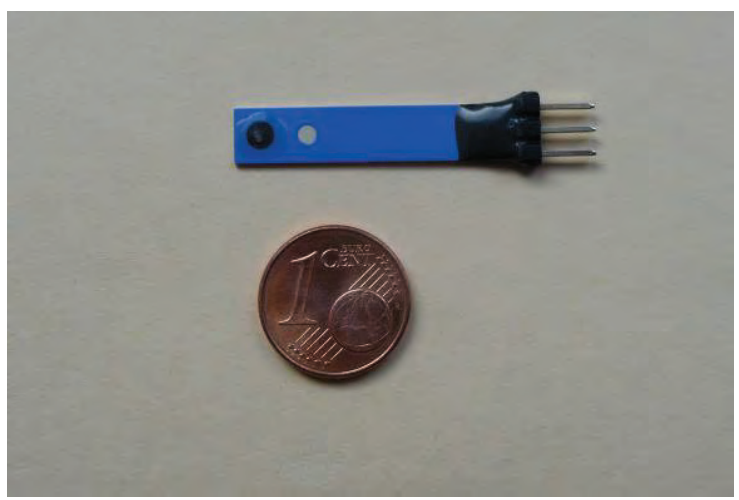

Abb.1 Potentiometrischer All-Solid-StateSensor

Dendritische Glycopolymere auf Basis von hochverzweigten Polyethyleniminen (PEI) mit Maltosestrukturen wurden synthetisiert und auf die Transducer-Oberflächen aufgebracht.

Abbildung 2 zeigt eine rasterelektronenmikroskopische Aufnahme (REM) einer mit 
einem dendritischen Polymer beschichteten, graphithaltigen Dickschichtelektrode.

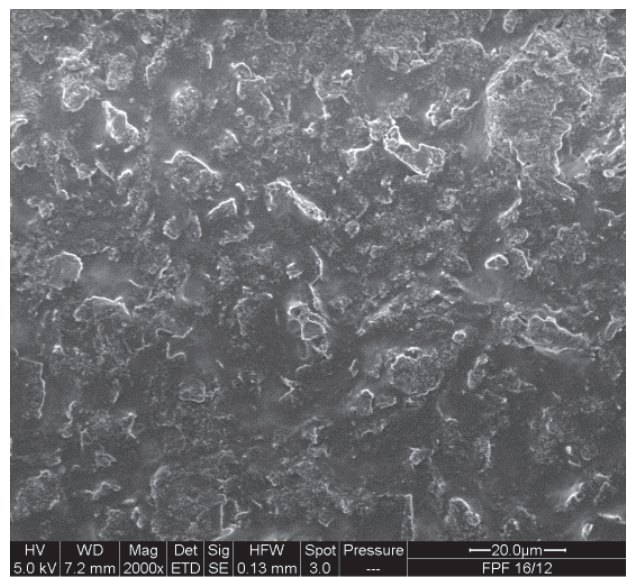

Abb.2 REM- Aufnahme einer polymermodifizierten Elektrodenoberfläche

Abbildung 3 zeigt das potentiometrische Ansprechverhalten einer $\mathrm{NH}_{4}^{+}-(a)$, einer $\mathrm{Ca}^{2+}$ (b), und einer $\mathrm{Pb}^{2+}$ - selektiven All-Solid-StateElektrode (c) in Lösungen verschiedener lonenkonzentrationen. Die potentiometrischen Untersuchungen erfolgten mit konventionellen $\mathrm{Ag} / \mathrm{AgCl}-$ Referenzelektroden. Die Steilheiten betragen in den untersuchten Konzentrationsbereichen durchschnittlich > $25 \mathrm{mV} /$ Dekade.

Eine neu synthetisierte $\mathrm{Ca}^{2+}$-selektive Verbindung mit Calixaren-Struktur kam zur Anwendung. Gegenwärtig erfolgt die Synthese weiterer neuer kationenselektiver lonophore, die an dendritische Polymere angekoppelt werden.

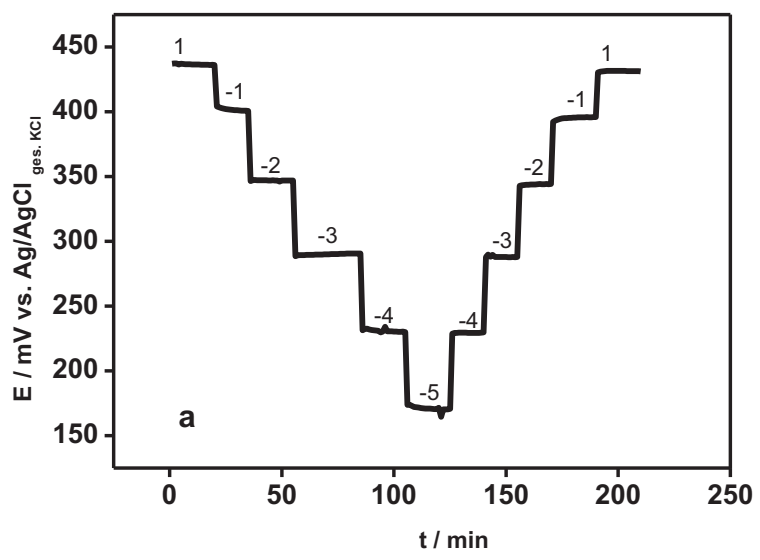

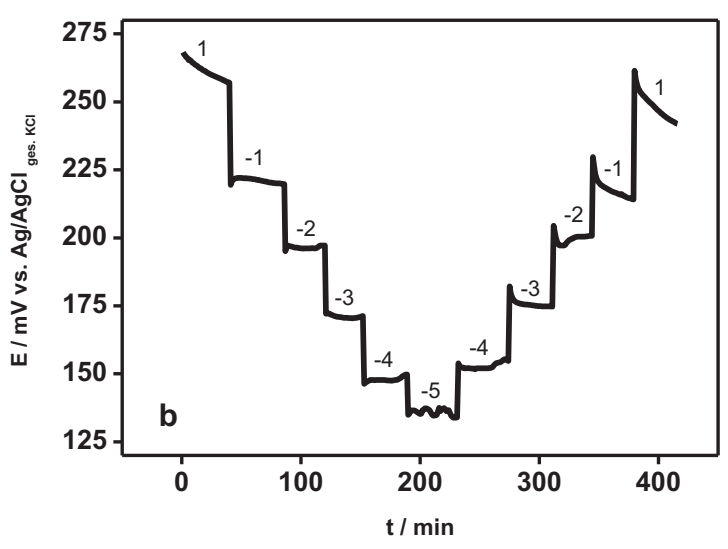

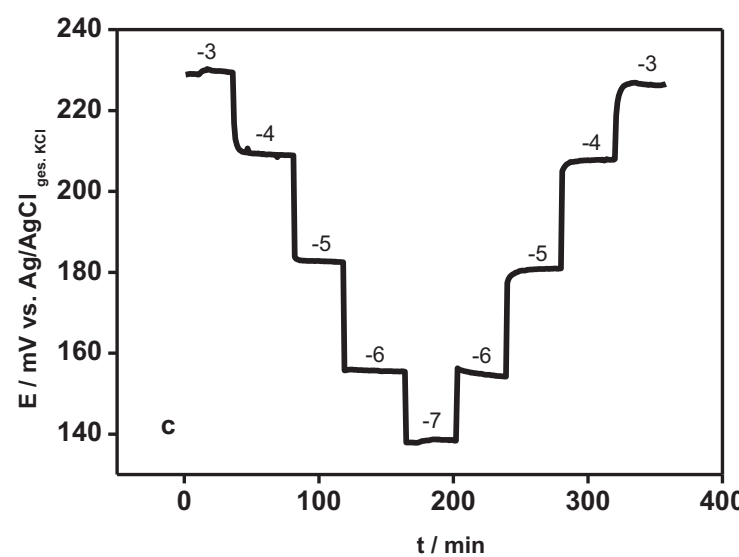

Abb.3 Potentiometrisches Ansprechverhalten von kationenselektiven Elektroden

a) $\mathrm{NH}_{4}^{+}$-selektive Elektrode

b) $\mathrm{Ca}^{2+}$-selektive Elektrode

c) $\mathrm{Pb}^{2+}$-selektive Elektrode

\section{Zusammenfassung und Ausblick}

Ionenselektive All-Solid-State-Elektroden auf Basis polymerhaltiger funktionaler Schichten wurden in Kombination mit siebdruckgefertigten Referenzelektroden zur potentiometrischen lonen-Bestimmung eingesetzt. Mit den ionenselektiven All-SolidState-Elektroden stehen leicht handhabbare, Vor-Ort einsetzbare Analysatoren zur Verfügung. Die Synthese neuer lonophore, die in dendritische Polymere eingebunden sind, steht zukünftig im Vordergrund der Arbeiten. Die aus mehreren funktionalen Schichten bestehenden Elektroden ermöglichen eine reproduzierbare und kostengünstige Elektrodenherstellung.

\section{Literatur}

[1] J. Bobacka, A. Ivanka, A. Lewenstam, "Potentiometric Ion Sensors", Chem. Rev. 2008, 108, 329-351

[2] K. Y. Chumbinumi-Torres, N. Rubinova, A. Radu, L. T. Kubota, E. 
Bakker, "Solid Contact Potentiometric Sensors for Trace Level Measurements", Anal. Chem. 2006, 15, 1318-1322

[3] E. Bakker, V. Bhahthavatsalan, K. L. Gemene, "Beyond Potentiometry: Robust Electrochemical Ion Sensor, Concepts in View of Remote Chemical Sensing", Talanta, 2008, 15, 629-635

[4] J. Bobacka, "Conducting Polymerbased Solid-State-Ion-Selective Electrodes", Electroanalysis, 18 (2006), 7-18

[5] J. E. Zachara, R. Toczylowska, R. Pokrop, M. Zagorska, A. Dybko, W. Wroblewski, "Miniaturised All-SolidState Potentiometric Ion Sensors based on PVC-Membranes containing Conducting Polymers", Sensors and Actuators B 101 (2004), 207-212

\section{Dank}

Die Autoren danken der Sächsischen Aufbaubank (SAB) für die finanzielle Unterstützung des Vorhabens (Förderkennzeichen: 4-753.50-03-84312/1). 